Slavica

bruxellensia

\section{Slavica bruxellensia}

Revue polyphonique de littérature, culture et histoire

slaves

$8 \mid 2012$

Migration(s) et Exil(s)

\title{
Andrej Tarkovskij en France : le dernier exil européen du cinéaste
}

\section{Nicolas Planchard}

\section{OpenEdition \\ Journals}

Édition électronique

URL : http://journals.openedition.org/slavica/1080

DOI : 10.4000/slavica. 1080

ISSN : 2034-6395

\section{Éditeur}

Université libre de Bruxelles - ULB

\section{Référence électronique}

Nicolas Planchard, «Andrej Tarkovskij en France : le dernier exil européen du cinéaste », Slavica bruxellensia [En ligne], 8 | 2012, mis en ligne le 15 juin 2012, consulté le 01 mai 2019. URL : http:// journals.openedition.org/slavica/1080 ; DOI : 10.4000/slavica.1080

Ce document a été généré automatiquement le 1 mai 2019.

\section{(c) $(1) \odot$}

Les contenus de Slavica bruxellensia sont mis à disposition selon les termes de la Licence Creative Commons Attribution - Pas d'Utilisation Commerciale - Pas de Modification 3.0 France. 


\title{
Andrej Tarkovskij en France : le dernier exil européen du cinéaste
}

\author{
Nicolas Planchard
}

1 Le lundi 5 janvier 1987, à l'issue des obsèques qui ont lieu à la cathédrale SaintAlexandre Nevski, rue Daru à Paris, le cinéaste russe Andrej Tarkovskij est inhumé au cimetière orthodoxe de SainteGeneviève-des-Bois ${ }^{1}$. Quelques années plus tard, Sergej Paradžanov s'étonnera que son ami et collègue repose en France, un pays où Tarkovskij n'avait pratiquement pas d'attache personnelle. Au début des années 1980, c'est l'Italie et la ville de Florence que

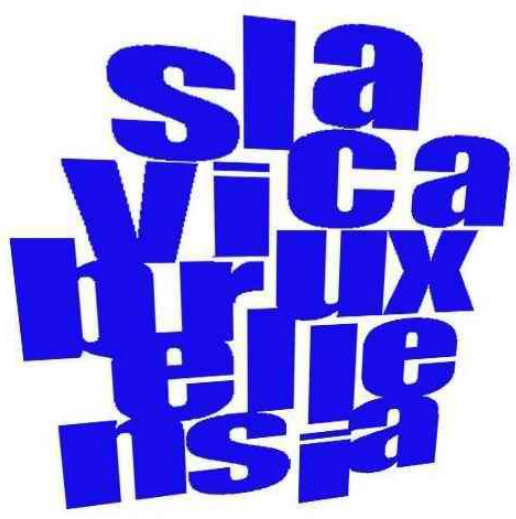
le réalisateur avait choisi pour son exil européen. C'était un départ redouté mais que le cinéaste jugeait inévitable pour la poursuite de sa carrière. Si les films de Tarkovskij étaient produits (non sans générosité par l'URSS) au sein du Goskino, l'équivalent d'un ministère du cinéma, la reconnaissance de son œuvre se fit principalement en Europe.

2 Tarkovskij aimait le cinéma de Robert Bresson et de Jean Vigo mais il connaissait peu la France. Malgré la multitudes de contacts professionnels établis depuis les années 1960, la France sera surtout une terre de souffrances et d'agonie, liée au cancer qui ronge l'artiste durant l'année 1986. La France a néanmoins joué un rôle déterminant dans la reconnaissance de ses talents. Les premiers soutiens occidentaux de Tarkovskij sont italiens et français. Tandis qu'en septembre 1962, le festival de Venise couronne L'Enfance d'Ivan (Lion d'or ex cequo avec le Journal Intime de Valerio Zurlini), c'est Jean-Paul Sartre en personne qui défend publiquement ce premier film face aux accusations de la presse communiste italienne ${ }^{2}$. Quand L'Unita déplore l'aspect surréaliste de certaines scènes, Sartre justifie l'outrance et le dépassement de la réalité. En raison de leur proximité 
idéologique avec l'URSS, les médias communistes français publient d'excellentes critiques du film (L'Humanités $e^{3}$, Les Lettres Françaises ${ }^{4}$ ). Ces journalistes assurent aimer L'Enfance d'Ivan parce que c'est un bon film mais ils semblent surtout apprécier en celui-ci un bon film soviétique. À Venise, la plupart des journalistes sont favorables au premier longmétrage de Tarkovskij mais certains soupçonnent L'Enfance d'Ivan d'avoir été récompensé pour des raisons diplomatiques ${ }^{5}$. La presse aime cette première œuvre pessimiste et jugée originale par rapport aux canons de l'académisme du cinéma soviétique. Dès son premier film, Tarkovskij suscite l'intérêt des festivals et des cercles communistes occidentaux. En URSS, cette reconnaissance précoce va constituer un formidable écran protecteur lorsque les dirigeants soviétiques signeront la fin du dégel cinématographique.

3 En mai 1969, Andreï Roublev, le second long-métrage de Tarkovskij, parvient au festival de Cannes. Le contexte s'avère bien différent : retenu pendant plus de deux années, censuré à domicile, le film est tenu à l'écart de la compétition officielle en raison de la réprobation soviétique. Mal aimé à Moscou, Andrei Roublev doit sa présence cannoise à la détermination de Robert Fabre le Bret, le secrétaire général du festival, lui-même soutenu par le ministre des Affaires culturelles André Malraux et grâce aux soins d'Otar Teneichvil de Sovexport ${ }^{6}$. À Cannes, le film bouleverse les journalistes qui vantent un authentique " chef-d'œuvre " ${ }^{7}$. Les critiques présents à Cannes sont sensibles à cet hymne à la liberté artistique venu d'URSS, cette fresque médiévale à la fois brulante et spirituelle, très éloignée de l'académisme qui mine alors les productions Mosfilm. Les soupçons de censure sont confirmés par la rocambolesque tentative soviétique de stopper la distribution du film à Paris en novembre 1969. Malgré des mises en gardes officielles venues de Moscou, le distributeur français Boris Gourevitch parvient à projeter le film sur les Champs-Élysées ${ }^{8}$ avec la complicité du ciné-club de L'Humanité ${ }^{9}$. Au bout du compte, ces tracasseries constituent une très bonne publicité pour Tarkovskij dans un contexte critique exceptionnel. Depuis la sortie française d'Andreï Roublev, la presse française compare non seulement Tarkovskij à Sergej M. Ejzenštejn mais aussi à Aleksandr Solženicyn. Contrairement à L'Enfance d'Ivan, plus personne ne conteste le talent de Tarkovskij et la dissidence de son œuvre est désormais envisagée. Isolé en URSS dans un climat de censure qui clôt le "dégel», ces soutiens occidentaux constituent une importante protection médiatique. Malgré les difficultés qu'il endure en URSS, Tarkovskij se sait apprécié et soutenu en Europe.

4 Le festival de Cannes confirme son attachement au cinéma de Tarkovskij avec le Grand prix spécial du Jury attribué à Solaris en mai 1972. À la différence des polémiques de l'affaire Andreï Roublev, il s'agit d'une participation très officielle: Tarkovskij et la comédienne NataLja Bondartčuk accompagnent le film en France. Cinéaste soviétique à la thématique spirituelle, Tarkovskij est à la fois soutenu par la presse communiste ( L'Humanité) et par les médias d'obédience catholique (Télérama). Considéré comme le principal réalisateur d'URSS en activité, il n'est toutefois pas autorisé à envoyer Le Miroir au festival de Cannes entre 1974 et 1975. Publiquement méprisé en URSS, Le Miroir est tardivement distribué par Gaumont qui ambitionne sous l'impulsion de Daniel Toscan du Plantier de démocratiser les grands cinéastes européens. En 1978, la participation du cinéaste au journal télévisé de TF1 contraste avec les condamnations officielles dont Le Miroir fait l'objet en URSS. Loin des circuits marginaux d'arts et d'essai où les autorités soviétiques le cantonnent, Le Miroir est à l'affiche de l'une des plus prestigieuses salles des Champs-Elysées. Tout au long des années 1970, Tarkovskij conforte son aura parmi la presse et les distributeurs français. En 1979, écarté de Cannes par le Goskino au profit de 
Sibériade d'Andrej Končalovskij, Stalker est également indisponible pour le festival de Venise. Fatigué de ces tracas récurrents, Tarkovskij envisage à la fin des années 1970 de partir travailler en Italie. Avec Stalker, sa carrière soviétique s'achève. Venise et Cannes ont révélé les talents de Tarkovskij et après plus d'une décennie de productions généreuses mais contrariées par le Goskino le cinéaste n'envisage plus son avenir professionnel qu'en Occident.

5 Malgré les soupçons du Goskino, Tarkovskij parvient à faire valider plusieurs mois de résidences italiennes consacrées à l'écriture de Nostalghia auprès du scénariste Tonino Guerra. En 1980, après la relégation soviétique du film en troisième catégorie, rien n'indique que Stalker pourra concourir dans les festivals de cinéma européens d'autant que le long-métrage tarde à sortir à Moscou. À Cannes, malgré les réticences soviétiques, plusieurs soutiens français se mobilisent pour présenter Stalker. Après les appuis de Robert Fabre le Bret et de Boris Gourevitch en 1969, ce sont le délégué général du festival Gilles Jacob et le distributeur Richard Delmotte de la société Audiphone ${ }^{10}$ qui organisent une projection surprise le 13 mai 1980. En dépit de conditions de projections semiclandestines et perturbées par des pannes d'électricité, Stalker hypnotise les journalistes ${ }^{11}$ . Le cinquième film de Tarkovskij obtient logiquement le Prix de la critique française. Médiatiquement, Stalker installe Tarkovski comme l'un des cinéastes les plus talentueux des années 1980.

6 Les bénéfices de ce concert de louanges sont évidents: la production italienne de Nostalghia s'accélère tandisque Gaumont rachète tous les longs métrages soviétiques de Tarkovskij au distributeur Audiphone. De surcroît, cette riche médiatisation occidentale permet à Stalker de quitter son purgatoire soviétique : contre toute attente, le film sort à Moscou le 19 mai 1980. Avec la complicité de Claude Philippot qui assure la distribution française de Stalker pour Gaumont, en novembre 1981, Serge Daney rapporte dans Libération les mauvaises manières de l'industrie cinématographique soviétique ${ }^{12}$. Le critique révèle que le cinéaste n'est pas autorisé à assister à la première parisienne de son film. Depuis ses séjours suédois et londoniens au début des années 1980, les responsables de l'URSS doutent de la loyauté de Tarkovskij et cherchent à éviter toute fuite à l'Ouest.

7 Un temps conciliant avec le Goskino, le cinéaste s'envole enfin pour Rome en mars 1982 afin d'y tourner Nostalghia pour la RAI, en partenariat avec Gaumont. Il ne reverra jamais l'URSS. Afin de prévenir toute fuite des époux Tarkovskij, les autorités n'accordent pas de visa à leur fils cadet qui reste à Moscou. Andrej et sa femme Larisa sont censés revenir en URSS à l'issue de ce tournage italien. Craignant de ne plus pouvoir repartir en Europe s'il retourne à Moscou, le cinéaste fait tout pour prolonger ses résidences occidentales. Il ajourne même le tournage des séquences russes de Nostalghia qui sont finalement réalisées en Italie. Lorsque Tarkovski rencontre Gilles Jacob à Rome en mars 1982, le réalisateur suggère une rencontre avec un membre du gouvernement français afin de faciliter la venue de son fils enEurope. Déjà, le départ de Tarkovskij parait définitif. En mai 1983, le cinéaste soumet son sixième long-métrage au jury du trente-cinquième festival de Cannes. Ayant acquis une certaine autonomie artistique grâce au Lion d'or obtenu vingt ans plus tôt, le réalisateur espère probablement obtenir une Palme d'or synonyme de liberté pour lui et les siens. Plongée au cœur de l'agitation de Cannes, la profonde mélancolie de Nostalghia divise les festivaliers et n'obtient qu'un lot de consolation, créé sur mesure pour Tarkovskij et Bresson : le Prix du cinéma de création. À l'été 1983, malgré les mises en gardes soviétiques, les époux Tarkovskij refusent de rentrer en URSS. Le cinéaste craint de ne plus pouvoir travailler librement à Moscou. Il 
demeure auprès de ses soutiens professionnels qui sont italiens, suédois (production du Sacrifice) et français.

Après le temps des résidences occidentales autorisées par le Goskino, l'heure est à des tractations difficiles. Le cinéaste multiplie les courriers adressés aux dirigeants soviétiques pour que son fils le rejoigne en Europe. Pour toute réponse, il se voit signifier un retour impératif à Moscou. Lors d'une rétrospective Tarkovskij organisée par Gaumont en avril 1984, certains journalistes français s'interrogent sur le mystérieux exil de l'artiste soviétique. L'Humanité fustige la politisation du «cas Tarkovski» et les polémiques redoublent ${ }^{13}$. De son côté, le réalisateur se désole de vivre séparé d'un fils qu'il n'a pas revu depuis près de deux ans. Encouragé par le violoncelliste Mstislav Rostropovič, un autre talent soviétique en exil, le cinéaste constate que ses réclamations diplomatiques ne débouchent sur rien. Il est temps d'impliquer les médias pour faire plier les dirigeants d'URSS. Dans un climat de Guerre froide symbolisé par le boycott des Jeux Olympiques de Los Angeles par les délégations soviétiques, Tarkovskij annonce qu'il demande l'asile politique aux États-Unis lors d'une conférence de presse organisée le 10 juillet 1984 au siège du Movimento Popolare à Milan par le député européen Roberto Formigoni. Le cinéaste prend les occidentaux à témoin: il dénonce ses conditions de travail soviétiques et exige la venue de son fils. Les journalistes français rapportent les souffrances d'un artiste spirituel qui assure ne pas être un dissident : "Dans ma vie, j'ai vécu des moments difficiles. Aujourd'hui, c'est le moment le plus dramatique de tous... Je suis forcé de quitter mon pays. $»^{14}$ À Milan, le cinéaste est entouré de Rostropovič, de l'écrivain Vladimir Maksimov et du metteur en scène Jurij Ljubimov, tout juste déchu de la citoyenneté soviétique. Par leur présence à la conférence de presse, ces trois talents valident les déclarations de Tarkovskij sur l'art en URSS. Tout en mobilisant les occidentaux, le cinéaste évite de trop brusquer les dirigeants soviétiques. Le scandale ne doit pas menacer ses proches demeurés en URSS. À Milan, le metteur en scène le répète aux journalistes : son avenir professionnel est à l'Ouest, aux côtés de ses producteurs. Le refus de Tarkovskij de rentrer à Moscou s'inscrit dans un contexte d'hémorragie des talents soviétiques. Le cinéaste a beau clamer un apolitisme sincère, médiatiquement, sa démarche contribue au dénigrement occidental de l'Union soviétique.

Aux journalistes européens, Tarkovskij annonce la réalisation, en Suède, d'un film inspiré par l'épreuve de l'exil: Le Sacrifice. De nouveaux soutiens s'activent pour qu'«Andrioucha» rejoigne ses parents en Europe. Les silences des Soviétiques contraignent Tarkovskij à prendre à nouveau la parole en décembre 1984 à Paris lors d'une seconde conférence de presse. En attendant que Gaumont débloque la sortie française de Nostalghia, retardée par le naufrage de Gaumont Italie, le distributeur Claude Philippot et le journaliste Robert Chazal mobilisent les médias avec la création d'un comité de soutien. Ces appuis français observent les réserves du cinéaste pour les affaires politiques : «Il était prudent et ne souhaitait pas être récupéré. ${ }^{15}$ Voilà près de trois ans que le cinéaste n'a pas revu son fils. Les mois qui passent attisent sa douleur : il critique ouvertement les dirigeants du cinéma soviétique. Bénéficiant de la ferveur d'un réseau de journalistes et de distributeurs occidentaux, l'artiste peut compter sur des soutiens fidèles dans le combat engagé pour faire sortir son fils d'URSS. Si le temps de la Nostalghia voit l'éclosion de projets inespérés, les premières mobilisations n'ont pas fait plier Moscou. Pour Tarkovskij, l'heure est à la réalisation d'un Sacrifice tristement prémonitoire. 
10 Pendant près d'une année et demie, Tarkovskij maintient la ligne défensive adoptée lors de la conférence de presse de Milan : l'exil lui a été imposé par le Goskino et c'est à regret qu'il est parti travailler à l'Ouest pour se rapprocher géographiquement de ses producteurs. Le battage entrepris est incomplet : l'artiste milite tardivement, à reculons, avec une prudence apolitique qui dessert ses intérêts. Constatant à Paris l'inflexibilité de Mikhajl S. Gorbačëv sur les questions humanitaires, le cinéaste doit radicaliser ses démarches. En novembre 1985, Tarkovskij n'a plus rien à perdre: il a déjà perdu le bras de fer l'opposant à Moscou. Redoutant un grave cancer (qui lui sera confirmé quelques jours plus tard à Stockholm), il prend la parole aux Dossiers de l'écran, lors d'une émission consacrée à Andrej Sakharov et à la dissidence soviétique. Pour la première fois de sa carrière, le cinéaste affronte ouvertement les dirigeants de l'URSS. Tarkovskij explose en direct à la télévision française en dénonçant l'inhumanité de Gorbačëv. L'influence de ce battage médiatique est difficile à mesurer. Certes, la sécession du réalisateur date de 1984 mais jamais son opposition n'avait pris une telle ampleur. Peut-être son état de santé déclinant l'encourage-t-il à brusquer ses démarches. Tandis que les autorités soviétiques font obstruction à la réunion de sa famille, le cinéaste craint que le temps ne joue contre lui. Ce que chacun ignore alors, c'est que les jours de Tarkovskij sont comptés. Tandis qu'il prévient les spectateurs qu'il risque de mourir sans son fils, il s'agit déjà de son ultime participation à une émission de télévision.

11 À la fin de l'année 1985, un cancer est diagnostiqué au cinéaste. François Mitterrand fait alors pression sur le Secrétaire général du PCUS pour qu'« Andrioucha » et sa grand-mère rejoignent l'artiste que l'on craint condamné par la maladie. C'est à Paris, le 19 janvier 1986 qu'a lieu la réunion de la famille Tarkovskij, auprès du cancérologue Léon Schwartzenberg et de la comédienne Marina Vlady. Ce n'est pas l'issue heureuse et italienne que l'artiste avait espéré. S'il trouve à Paris soutiens politiques et soins médicaux, la France est une terre amère pour Tarkovskij : après quatre années de lutte pour faire sortir son fils d'URSS, il se sait condamné par la maladie. Alors que le cinéaste profitait en Italie de rencontres et de séjours stimulants, la France est une terre de repli, de soins, puis d'agonie. Tarkovskij n'avait pas choisi la France, ce sont des circonstances dramatiques qu'il encourage à demeurer auprès de ses médecins. C'est plutôt l'Italie mystique et chrétienne de Nostalghia qu'il envisageait comme terre d'accueil européenne. C'est d'ailleurs cette Italie brumeuse et spirituelle qu'il avait mis en scène dans Nostalghia. À Paris, un cercle d'amis se constitue avecMarina Vlady et Léon Schwartzenberg, Andrej Jablonskij, Vivianne Končalovskij, le producteur Anatole Dauman, le réalisateur Chris Marker, l'interprète et journaliste Victor Loupan et les soutiens sincères qu'offrent Charles de Brantes et Alain Malraux. Cette sollicitude touche Tarkovskij : «Que ferionsnous sans nos amis de maintenant? Le meilleur moyen de garder ses amis est de ne pas les trahir. $»^{16}$ La gratitude de l'artiste envers les appuis trouvés en France, en Italie et auprès des Russes exilés (Maksimov, Rostropovič, Maksim Šostakovič) peut s'expliquer par son amertume envers certains compatriotes :

12 À lire les confidences du Journal, les années de tourments et la séparation de sa famille ont laissé des blessures profondes.

13 À la suite du tournage scandinave du Sacrifice, le cinéaste avait prévu de s'installer à Florence, dans un appartement prêté par la municipalité de la ville. Si entre 1982 et 1986, le cinéaste ne cesse de voyager au gré de ses appuis européens, entre l'Italie, la RFA, la Suède et la France, il revendique toujours son identité artistique russe. Malgré les aléas de l'exil, son œuvre ne connaît pas de rupture. Qu'il travaille au Sud ou au Nord de l'Europe, 
le réalisateur maintient sa loyauté envers la mère patrie : "Je suis russe et je resterai russe. Même si je fais un film en Suède avec des acteurs suédois, ce sera quand même un film russe. Il y a là du Dostoïevski et du Soljenitsyne mais aussi quelque chose du Quichotte de Cervantès. $»^{19}$ Quand Le Sacrifice concourt à Cannes en mai 1986, les journalistes sont frappés par l'atmosphère dramatique d'une menace nucléaire qui renvoie aux sinistres nouvelles venues de Tchernobyl. Le long-métrage emporte l'adhésion des journalistes mais échoue à obtenir la Palme d'or, attribuée à Mission de Rolland Joffé. Signe de la gravité de l'état de santé de Tarkovskij, l'artiste ne peut accompagner son film à Cannes et c'est son fils qui reçoit pour lui le Grand prix du jury. Le Sacrifice domine la presse cinématographique et obtient des scores plus qu'honorables dans les salles françaises. De Stalker au Sacrifice en passant par Nostalghia, l'engouement des critiques pour le cinéma de Tarkovskij se maintient durant les années 1980. Malgré des difficultés permanentes, le réalisateur est un artiste de son temps et ne constitue pas un créateur maudit : son œuvre est admirée par les journalistes, ses films sont distingués dans les festivals de cinéma et il a bénéficié de comités de soutiens durant la bataille engagée pour faire sortir son fils d'URSS.

Depuis la réunion de la famille Tarkovskij à Paris en janvier 1986, la médiatisation de sa carrière est strictement cinématographique: les polémiques politiques sont refermées. Dès le festival de Cannes, certains critiques envisagent Le Sacrifice comme une œuvre testament ${ }^{20}$. La maladie qui épuise l'artiste lui interdit de rencontrer les journalistes. Si Le Sacrifice domine alors la presse cinématographique, l'année 1986 constitue une période de retrait médiatique, ponctuée de rares entretiens donnés à des personnes de confiance comme de Brantes. Durant les premiers mois de l'année 1986, Tarkovskij endure le lourd traitement des chimiothérapies etdes radiothérapies que supervise le professeur Schwartzenberg. Le Journal rapporte des soins particulièrement éprouvants mais l'artiste s'y exprime alors abondamment. Dans ce document personnel, Tarkovski approfondit ses réflexions sur ses réalisateurs de prédilection (Bresson, Ingmar Bergman, Federico Fellini) et envisage différents projets de films. Il évoque également les soutiens rencontrés en Occident, la déception venue d'anciens amis soviétiques et une maladie dont il craint l'issue fatale. À l'inverse, dès la fin du mois d'octobre 1986, les pages du Journal se font plus inquiétantes car les entrées y sont plus rares. Tarkovskij est épuisé, ses forces l'abandonnent. En novembre, la santé du cinéaste se dégrade brutalement : il doit s'aliter et les médecins ne peuvent enrailler la progression du cancer. C'est dans la nuit du 28 au 29 décembre 1986 que Tarkovskij meurt à la Clinique Hartmann de Neuilly. À l'heure où le Goskino semble enfin se libéraliser et qu'éclosent les premiers fruits de la Perestroïka, le cinéma soviétique perd son plus grand réalisateur depuis Eisenstein.

Dans son Journal, Tarkovskij a exprimé de la gratitude envers les Français qui ont pris en charge ses soins et facilité la venue de son fils en Europe. Pour autant, l'artiste n'avait guère d'attaches personnelles avec la France. Sa terre d'adoption occidentale demeurait l'Italie où il résidait depuis 1982. Outre l'appartement offert par la mairie de Florence, le cinéaste y avait acquis un terrain à Roccalbegna en Toscane. Il comptait y faire construire une maison avec des vignes, un verger, des oliviers... Tarkovskij sera finalement enterré près de Paris, une ville qu'il connaissait peu, sinon en tant que malade, durant la dernière année de sa vie, lorsqu'il était logé au numéro 10 de la rue Puvis de Chavannes, chez son producteur A. Dauman. La fidélité des soutiens français à Tarkovskij s'observe à la mort de l'artiste à Paris, le 29 décembre 1986. Les nombreux articles qui lui rendent hommage témoignent de l'important soutien médiatique qui a porté sa carrière en France ${ }^{21}$. 
16 La cérémonie du 5 janvier 1987 réunit la famille de l'artiste, ses proches et les appuis cinématographiques des années 1980. Au premier rang se tiennent Larisa Tarkovskaja et les deux fils du cinéaste, Andrej et Arsenij ${ }^{22}$, accompagnés par Olga ${ }^{23}$ et par la mère de Larisa. Autour du cercueil de Tarkovskij, les différents soutiens français lui rendent un ultime hommage. On y retrouve les relais humanitaires (Philippot, de Brantes), médiatiques (Marie-Noëlle Tranchant) ainsi que les coproducteurs européens de ses derniers films. Sont présents Toscan du Plantier (Gaumont), Jérôme Clément (CNC) et Dauman (Argos). Les obsèques réunissent également les nombreuses personnalités du septième art ayant croisé la route de Tarkovskij : Robert Bresson, Marina Vlady, Valérie Mairesse, Otar Iosseliani mais aussi le pianiste et chef d'orchestre Daniel Barenboïm. Naturellement, les exilés soviétiques sont présents à l'église russe de Paris: il y a Rostropovič et son épouse Galina Višnevskaja ainsi que l'écrivain Vl. Maksimov. Au nom du gouvernement, le Secrétaire d'État à la culture Philippe de Villiers présente ses condoléances à la famille. L'AFP rapporte l'affluence du public à la cérémonie : « Plusieurs centaines de personnes, (...) beaucoup de jeunes et d'anonymes. ${ }^{24}$ L'AFP ne mentionne pas la présence de représentants du gouvernement de l'URSS mais, sous la coupole de la cathédrale, des gerbes de fleurs parmi lesquelles celles du Goskino, de l'Union des cinéastes, de l'ambassadeur d'URSS, ainsi que «des petits bouquets anonymes ${ }^{25}$ entourent le cercueil de Tarkovskij. Le nombre de célébrités culturelles, comme les officiels français présents aux obsèques conforte l'idée que l'artiste n'était pas isolé. À la mort de Tarkovskij, Daney insiste dans Libération sur les multiples difficultés endurées en URSS mais il relève aussi tous les soutiens rencontrés en Europe ${ }^{26}$. Sans être populaires, les films de Tarkovskij avaient leur public à Paris. La présence de nombreux anonymes à la cathédrale Saint Alexandre Nevski survient quelques mois après le succès du Sacrifice au Box-office parisien.

17 Une dépêche de l'AFP détaille les obsèques du réalisateur. L'office religieux débute à 14 heures par un hommage rendu par le cosmopolite Boris Bobrinskoi :

Ces dernières paroles renvoient à la séquence de crucifixion d'Andreï Roublev, lorsque l'épisode biblique se mêle à l'histoire médiévale. Selon les rites russes, l'office est célébré à la lueur de petites bougies puis le cercueil est transporté dans la cour de la cathédrale. Deux compagnons d'exils rendent un dernier hommage à Tarkovskij. Maksimov, l'écrivain et rédacteur en chef de la revue Continent, qui jusqu'aux derniers jours de la maladie avait rendu visite au cinéaste à Paris, prononce une allocution. Ensuite, Rostropovič interprète, du haut des marches de l'église, un court morceau de Bach à la mémoire de son ami.

19 Cet épisode marque les personnes présentes à la cathédrale orthodoxe : « Rostropovič a joué face au cercueil, dans la cour. C'était simple et chargé de symboles $»^{28}$, se souvient Claude Philippot. D'une certaine manière, la Suite de Bach interprétée par le violoncelliste en exil préfigure les images qu'enregistreront les télévisions à la chute du Mur de Berlin en novembre 1989. Dans Le Figaro, Marie-Noëlle Tranchant revient sur le temps fort que constitue l'adieu du violoncelliste à Tarkovskij :

De Paris à Cannes en passant par Venise, la médiatisation dont le réalisateur a bénéficié de 1962 à 1987 constitue un exemple de mondialisation protectrice. De ce point de vue, il n'est pas anormal que Tarkovskij soit enterré à Paris. Du travelling vertical sur l'arbre et l'enfant qui ouvrait L'Enfance d'Ivan au dernier plan d'un enfant adossé à l'arbuste qui clôt Le Sacrifice, l'œuvre de Tarkovskij peut être envisagée comme une boucle spirituelle et mystique. À Paris, une autre boucle se referme : en novembre 1963, L'Enfance d'Ivan était 
projeté au Bonaparte de Jean-Paul Thirriot. Vingt-quatre ans après, c'est cette même salle qui projette encore Le Sacrifice, en cette journée du lundi 5 janvier 1987, le jour même des obsèques parisiennes de Tarkovskij ${ }^{30}$.

\section{NOTES}

1. Tranchant M.-N., «L'adieu à Tarkovski », in : Le Figaro, 6 janvier 1987.

2. Sartre J.-P., « Discussion sur la critique à propos de L'Enfance d'Ivan », in : Les Lettres françaises, 26 décembre 1963.

3. Lachize S., «L'Enfance assassinée », in : L'Humanité, 20 novembre 1963.

4. Sadoul G., « La vérité sur les rapports humains », in : Les Lettres Françaises, 14 novembre 1963.

5. de Baroncelli J, «L'Enfance d'Ivan », in : Le Monde, 12 novembre 1963.

6. Sovexport est chargée des échanges internationaux de films.

7. INA, « Le Masque et la Plume », radio France Inter, 8 juin 1969.

8. Billard P., «L'affaire Roublev », in : L'Express, $1^{\text {er }}$ décembre 1969.

9. Lachize S., « Andreï Roublev au ciné-club de l'Humanité », in : L'Humanité, 5 novembre 1969.

10. À Paris, la société de distribution Audiphone est chargée de l'échange des films avec l'URSS.

11. Murat P., "Tarkovski nous ouvre la chambre du bonheur ", in: Télérama, n 1585, 28 mai 1980.

12. Daney S., «L'autre zone », in : Libération, 20 novembre 1981.

13. Siclier J., "Le cas Tarkovski », in : Le Monde, 26 février 1984.

14. INA, Journal télévisé d'Antenne 2, présenté par Victor Vramant, diffusé le 10 juillet 1984.

15. Entretien de l'auteur avec Claude Philippot réalisé à Paris le 10 juillet 2007.

16. Tarkovski A., Journal, Cahiers du cinéma, Paris, 1993, p. 399.

17. Ibid., p. 414.

18. Ibid., p. 437.

19. Zand N., « Une villa tchékhovienne en Suède ", in : Le Monde, $1^{\mathrm{er}}$ juin 1985 (entretien avec le réalisateur).

20. Barron J. \& Hugeux V., « Tarkovski, le secret de l'arbre sec », in : La Croix, 14 mai 1986.

21. Waintrop E., Baecque de A., Daney S., "Tarkovski, la mort dans l'âme », in : Libération, 30 décembre 1986.

22. Arsenij Tarkovskij, le fils ainé du cinéaste, né d'un premier mariage avec Irma Rausch.

23. Olga Kizilova, la fille de Larisa Tarkovskaja.

24. «Recueillement pour les obsèques du cinéaste Andreï Tarkovski à la cathédrale russe de Paris », Dépêche AFP du 5 janvier 1987.

25. Idem.

26. Waintrop E., Baecque de A., Daney S., art. cit.

27. "Recueillement pour les obsèques... ", dépêche AFP du 5 janvier 1987.

28. Entretien réalisé avec l'auteur à Paris le 10 juillet 2007.

29. Tranchant M.-N., "L'adieu à Tarkovski ", art. cit.

30. «Résultats Paris-Périphérie du 31 décembre 1986 au 6 janvier 1987 », in : Le Film Français, n 2123, 16 janvier 1987. 


\section{RÉSUMÉS}

Depuis le Lion d'or obtenu par L'Enfance d'Ivan à Venise en 1962, l'Europe médiatise les talents du cinéaste soviétique Andreï Tarkovski. De 1966 à 1986, c'est alors le festival de Cannes qui se mobilise en faveur de films mal aimés (Andreï Roublev, Stalker) par les dirigeants d'URSS. Si Andrej Tarkovskij incarne la figure cinématographique de l'artiste russe, attaché à sa terre (les bois du Miroir) et fier de ses racines spirituelles (le pouvoir des icônes), l'Europe constitue un territoire protecteur et hospitalier. Qu'il s'agisse de journalistes (Serge Daney pour Libération), d'institutions (festival de Cannes, Ministère français de la culture) ou de personnalités du cinéma (Daniel Toscan du Plantier chez Gaumont), l'artiste est non seulement reconnu mais surtout soutenu à l'Ouest. Malgré les amples budgets de ses films soviétiques, les pressions idéologiques épuisent Tarkovskij qui finit par s'installer en Italie au milieu des années 1980. Refusant de rentrer à Moscou, le cinéaste est désormais épaulé par des comités de soutiens dans son combat pour faire sortir son fils d'URSS. Affrontant un grave cancer aux premiers jours de l'année 1986, l'artiste est soigné à Paris tandis que les autorités françaises facilitent la venue de son fils. Le réalisateur avait peu d'attaches personnelles avec la France mais c'est à Paris, parmi ses soutiens et ceux qui le tenaient pour un cinéaste de tout premier plan que Tarkovskij est enterré aux premiers jours de l'année 1987.

\section{INDEX}

Index chronologique : communisme, Guerre froide, XXe siècle

Index géographique : France, Italie, URSS

Mots-clés : cinéma russe, cinéma soviétique, exil

\section{AUTEURS}

\section{NICOLAS PLANCHARD}

Docteur en Histoire culturelle, Université de Versailles Saint-Quentin-en-Yvelines, Centre d'Histoire culturelle des sociétés contemporaines 